\title{
BMJ Open Development of a self-reported reflective tool on advanced access to support primary healthcare providers: study protocol of a mixed-method research design using an e-Delphi survey
}

\author{
Mylaine Breton (D) , ${ }^{1}$ Isabelle Gaboury (1) , ${ }^{2}$ Maxime Sasseville, ${ }^{3}$ \\ Christine Beaulieu, ${ }^{4}$ Sabina Abou Malham (D) , ${ }^{5}$ Catherine Hudon (D) ,6 \\ Isabel Rodrigues, ${ }^{7}$ Lara Maillet (D) , ${ }^{8}$ Arnaud Duhoux, ${ }^{9}$ Nadia Deville-Stoetzel (D) , ${ }^{4}$ \\ Jeannie Haggerty ${ }^{10}$
}

To cite: Breton M, Gaboury I, Sasseville $\mathrm{M}$, et al.

Development of a self-reported reflective tool on advanced access to support primary healthcare providers: study protocol of a mixed-method research design using an eDelphi

survey. BMJ Open

2021;11:e046411. doi:10.1136/ bmjopen-2020-046411

- Prepublication history for this paper is available online. To view these files, please visit the journal online (http://dx.doi org/10.1136/bmjopen-2020046411).

Received 29 0ctober 2020 Accepted 19 October 2021

Check for updates

(c) Author(s) (or their employer(s)) 2021. Re-use permitted under CC BY-NC. No commercial re-use. See rights and permissions. Published by BMJ.

For numbered affiliations see end of article.

Correspondence to

Dr Mylaine Breton;

mylaine.breton@usherbrooke.ca

\section{ABSTRACT}

Introduction Timely access is one of the cornerstones of strong primary healthcare (PHC). New models to increase timely access have emerged across the world, including advanced access (AA). Recently in Quebec, Canada, the AA model has spread widely across the province. The model has largely been implemented by PHC professionals with important variations; however, a tool to assess their practice improvement within $\mathrm{AA}$ is lacking. The general objective of this study is to develop a self-reported online reflective tool that will guide PHC professionals' reflection on their individual $A A$ practice and formulation of recommendations for improvement. Specific objectives are: (1) operationalisation of the pillars and subpillars of AA; (2) development of a self-reported questionnaire; and (3) evaluation of the psychometrics.

Methods and analysis The pillars composing Murray's model of AA will first be reviewed in collaboration with PHC professional and stakeholders, patients and researchers in a face-to-face meeting, with the goal to establish consensus on the pillars and subpillars of AA. Leading from these definitions, items will be identified for evaluation through an e-Delphi consultation. Three rounds are planned in 2020-2021 with a group of 20-25 experts. A repository of recommendations on how to improve one's AA practice will be populated based on the literature and enriched by our experts throughout the consultation. Median and measures of dispersions will be used to evaluate agreement. The resulting tool will then be evaluated by PHC professionals for psychometrics in 2021-2022.

Ethics and dissemination The Centre Intégré de Santé et de Services Sociaux de la Montérégie-Centre Scientific Research Committee approved the protocol, and the Research Ethics Board provided ethics approval (2020-441, CP 980475). Dissemination plan is a mix of community diffusion through and for our partners and to the scientific community including peer-reviewed publications and conference presentations.
Strengths and limitations of this study

- Provides a revisited and operationalisation of the pillars and subpillars of the advanced access (AA) model developed 25 years ago.

- Provides a rigorously developed up-to-date tool on AA based on the literature and on the experiences of various primary healthcare stakeholders in response to their expressed needs.

- Involves the participation of multiple stakeholders with different roles and attached to diverse organisation originating from multiple environments including urban, rural and remote regions.

- The Delphi method allows the experts to express their thoughts independently, while encouraging pragmatism, honesty and creativity.

- The developed tool may not be transferable without cultural adaptation to other settings where the principles of AA are implemented.

\section{INTRODUCTION}

\section{Advanced access model}

Timely access is widely recognised as a cornerstone of effective primary healthcare (PHC). The advanced access (AA) model, developed to increase timely access, has been promoted and adopted in primary care settings in various countries. It is the most commonly used organisational model to reduce wait times for primary care appointments. ${ }^{1}$ Timely access is one of the guiding principles of the patient-centred medical home (PCMH) ${ }^{2}$ The pillars of AA complement the PCMH model (timely access, comprehensiveness, continuity, interprofessional collaboration) and emphasise organisational components. AA 


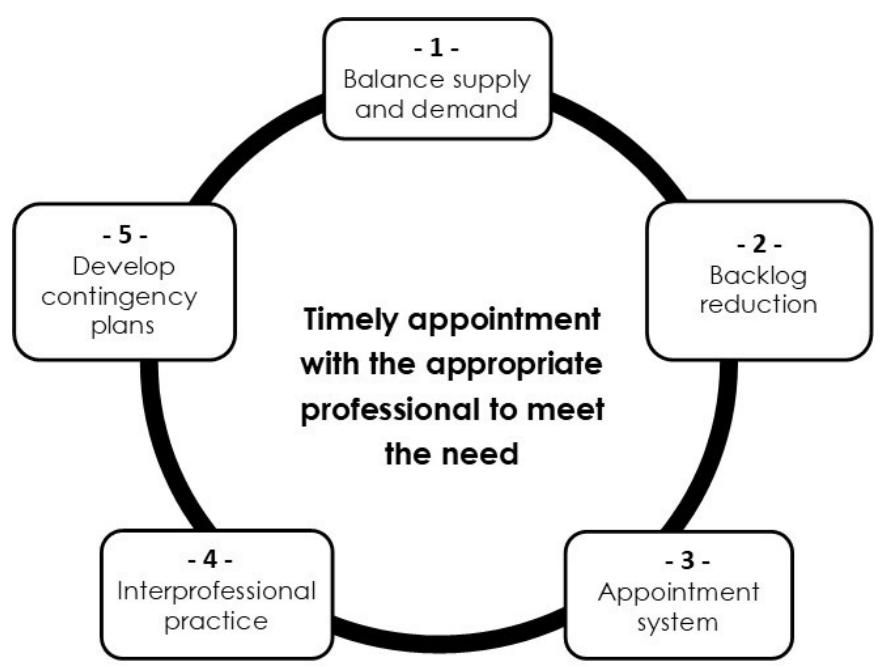

Figure 1 The five pillars of advanced access. The figure shows the advanced access model with the original five pillars and guiding principles. This is used as a starting point for this proposal.

has been defined according to five pillars ${ }^{34}$ : (1) balance supply and demand; (2) reduce the backlog of previously scheduled appointments; (3) review the appointment system; (4) integrate interprofessional practice; and (5) develop contingency plans (see figure 1). The AA model was developed in the USA in 2001 and implemented in North America and Europe, with many studies in the USA, the UK and Canada and its effectiveness demonstrated in various healthcare systems. ${ }^{5-9}$ Benefits of AA include reduced wait times, ${ }^{56}$ 9-11 fewer missed appointments $^{510}$ and improved professional and ${ }^{812}$ patient satisfaction, ${ }^{5}$ and provider productivity. ${ }^{9}$

The AA model aligns with the organisational guiding principles for a high-quality high-performance primary care organisation as put forward by the College of Family Physicians of Canada. ${ }^{13} 14$ That said, even if the concept of AA was developed more than 20 years ago by Murray et al remains current, it needs to be adapted to the contemporary context. ${ }^{15}$ This study contributes to refine AA based on more interdisciplinary-based team and the need to improve PHC practice with a quality improvement approach.

\section{Evolution of AA and state of research in the province of Quebec}

In Quebec, AA was first introduced in 2012, and since then it has been widely promoted by the Quebec College of Family Physicians, as well as by the Ministry of Health and Social Services. ${ }^{16} 17$ Family physicians are strongly encouraged to implement an AA model based on the five pillars proposed by Murray et $a l^{3}{ }^{3}$ Over the past 6 years, the majority of PHC family physicians in Quebec have introduced AA in their organisations at varying levels of implementation. ${ }^{418}$

\section{An expressed need for a reflective tool}

Several guides have been developed in Canada, ${ }^{18-20}$ the $\mathrm{USA}^{21-23}$ and Europe ${ }^{24}$ to assist PHC professionals and/ or organisations to plan and implement AA. These guides offer recommendations to plan supply, reduce demand and organise appointment management in order to achieve and maintain a balance between supply and demand, thus enabling timely responses to patient requests. The guides generally present principles of $\mathrm{AA}$, along with how to implement initial changes and some measurement tools. They also offer strategies to support the introduction of AA, but lack information and guidance to improve and sustain AA or to troubleshoot issues over time.

There is no tool even to evaluate the status of AA in a professional's practice, let alone to guide its continuous improvement and sustainability. Inspired by the principles of the Model for Improvement, ${ }^{25}$ such a tool could be used to align metrics across multiple PHC providers, while operationalising the complex process of providing access to care in a daily practice. One could use the developed tool to reflect on one's practice and plan for modifications to improve patient access. This project was therefore developed in response to a clearly expressed ministerial desire to meet the needs of PHC professionals to be supported in the clinical integration and improvement of AA in PHC settings. This study will provide an online reflective tool that will be used as requested by PHC professionals seeking to improve their AA practice.

Self-reported tools are useful reflective strategies to support quality improvement as they are easily accessible and available when needed, regardless of location. These tools also provide an effective way to promote selfreflection and identification of strengths and areas in need. ${ }^{26}$ Some tools available online, provide diagnosis, document or assess a level of practice or alignment with goals such as those of the tool developed by the College of Family Physicians in Canada to assess the $\mathrm{PCMH}^{27}$ or the Universal Health Coverage Primary Health Care SelfAssessment Tool. ${ }^{28}$ Online tools may also offer a reflective perspective to provide actionable advice, ${ }^{27}$ or immediate results and guidance. ${ }^{29}$

There is a need to develop a self-reported online reflective tool to support AA implementation and improvement by PHC providers. To achieve this objective, it is important to ensure that there is consensus on the underlying model. Differences in definition or interpretation of the pillars of AA could lead to operationalisation difficulties, that can and should be avoided.

\section{Objectives}

The main objective of this study is to develop a selfreported reflective tool to support PHC providers to improve their AA practice.

The specific objectives are to:

1. Operationalise the pillars and subpillars of the AA model.

2. Develop a self-reported questionnaire on their practice in AA.

i. Develop a questionnaire to assess the level of implementation of the AA model. 
ii. Identify key recommendations to improve AA.

3. Evaluate the psychometrics of the tool.

The main deliverable of our study will be a self-reported reflective tool on AA that will be combined with a repository of recommendations to improve AA, available on an electronic platform easily accessible to PHC providers and teams. This includes physicians, nurses and nurse practitioners, social workers, pharmacists, nutritionists, psychologists and so on. Users will receive, in one place, an evaluation of their AA practice and personalised recommendations to support improvement.

\section{METHODS}

\section{Study design overview}

A modified Delphi methodology will be used, to develop a reflective tool and identity strategies to improve AA. A literature review and analysis of selected articles will be used to identify conceptual constructs, followed by an iterative consensus achievement process among key experts including a face-to-face meeting and an online survey tool (e-Delphi). ${ }^{30}$ Using an iterative process, a Delphi consultation is an effective technique designed to obtain the most reliable consensus within a group of experts regardless of their geographical spread. ${ }^{31-33}$ A group of experts from the province of Québec will include diverse providers such as family physicians, nurse practitioners, nurses, front-desk and administrative staff and policymakers. Grounding the initial Delphi round in concepts derived from literature and based on initial experts' input during the face-to-face meeting will both be efficient, and will stimulate participation in the following steps of the tool development.

The process entails three sequential phases, with phase 1 being qualitative, while phases 2 and 3 being including quantitation. Table 1 briefly presents all three phases with their specific objectives. Phase 1 consists of a face-to-face meeting. This pre e-Delphi consultation aims to establish common bases in the operationalisation of a revised AA model (objective 1). Phase 2 will consist of a three-round consultation to identify the content of the self-reported reflective tool. Phase 3 will follow to assess the developed reflective tool and its applicability to different PHC professionals and in different PHC environments.

The self-reported reflective tool aims to evaluate the processes associated with each AA pillar while allowing its users to grasp their strengths and weaknesses with respect to their level of implementation.

\section{Study management}

The research team includes researchers with AA and methodological expertise, and PHC professionals including family physicians and nurses. The team will oversee the development and ongoing processes of the study, as well as major decisions regarding the selection of AA experts to invite to the face-to-face meeting (Phase 1) and to the e-Delphi consultation (Phase 2). They will also be involved in piloting material and instruments ahead of consultations.

\section{Table 1 The AA reflective tool development in brief}

\section{Phase 1}

Pre-Delphi consultation Establishing common bases

- Operationalise the AA model

\section{Phase 2}

e-Delphi consultation

Creation of the AA reflective tool

(list of essential items to be assessed in clinicians) the reflective tool by $\mathrm{PHC}$ practitioners/

- Research team identifies AA pillars and definitions from the literature

- Identification and recruitment of AA experts

- Consensus building on pillars and brainstorming about subpillars of AA through a facilitated face-to-face meeting

\section{First round of consultation}

- Panel expert agreements scores (from 1 to 9) on subpillars and definitions

- Suggestions/comments for modification or addition to subpillars

Second round of consultation

- Global and individual feedback report from round 1

- (Level of consensus achieved, global and individual expert panel scores)

- Panel expert agreement scores (from 1 to 9) on new propositions and modification emerging from round 1

- Panel expert agreement scores (from 1 to 5 ) on the importance of each subpillar and the list of items to measure their level of implementation

- Panel expert agreement (yes/no) on suggested response scales

- Suggestions/comments for modification or addition of items

Third round of consultation

- Global and individual feedback report from round 2

- (Level of consensus achieved, global and individual expert panel scores)

- Consensus building on items by pillar and subpillar

- Suggestions for practical recommendations by item to provide to clinicians to improve their AA practice

Phase 3

Piloting, adjustment and development of a repository of recommendations

To assess the tool and its applicability in different PHC environments
- Questionnaire completion by PHC clinicians in different PHC settings

- Psychometric properties analyses

- Focus groups to receive feedback and improvement tips

- Final adjustments

- Development of a repository of recommendations with actionable guidance 


\section{The expert panel}

To maximise the acceptability and usefulness of the reflective tool in Quebec's contemporary context, the research team will establish an AA expert panel comprised of provincial and local decision-makers, family physicians, practitioner and clinical nurses, continuous quality improvement officers, front-desk and administrative staff, as well as patients and researchers working in the field of AA. We will seek to bring together experts with diverse expertise based on their role in their own organisation as well as at the local, regional or provincial level. Purposive and snowball sampling techniques will be used to identify eligible participants. Forty potential participants will first be approached and invited by the principal investigators by email to join the expert panel and to be part of the pre-Delphi meeting with the hope of recruiting and maintaining a sample of 20-25 experts across all three e-Delphi rounds. This is above the target of 10-18 individuals, to ensure the development of productive group dynamics and to maximise chances of reaching consensus among experts. ${ }^{30}$ Participants will be considered for the panel if they are working in PHC or belong to an organisation working closely with PHC professionals, and have an extensive experience with AA (5+ years) as a practitioner or manager. Practitioners and managers who were involved in the development of the training sessions provided by the Quebec College of Family Physicians will also be invited.$^{34}$ Strategies to maximise the retention rate include personalised reminders from one of the principal investigators, with the goal of not losing more than $30 \%$ of the participants over the three expected rounds. ${ }^{30}$

\section{Phase 1: operationalisation of the AA model}

Building a consensus around the AA model will first entail agreeing on the pillars and subpillars that are essential to integrate in an AA practice in PHC. The starting point will be the conceptual framework of the five guiding principles of AA developed by Murray and Berwick in $2003^{3}$ : (1) balance supply and demand; (2) reduce the backlog of previously scheduled appointments; (3) review the appointment system; (4) integrate interprofessional practice; and (5) Develop contingency plans (see figure 1).

Phase 1 will consist of a literature review to conceptualise a revised AA model. Search terms such as 'advanced access,' 'open-access,' 'same-day scheduling,' 'timely access' and 'AA implementation' will be used. The literature review will include scientific studies and grey literature reports at local, national and international levels. This scan will allow us to delineate the pillars and subpillars as defined in models of AA in the contemporary literature, as well as constructs that need to be measured within each subpillar.

Following revision by the research team, the revised AA model will be submitted to the AA expert panel in a faceto-face meeting for discussions to build consensus while refining the pillars and definitions. This in-person meeting will be highly interactive and use facilitation techniques for group consultations, to encourage everyone's participation.
The meeting will be organised in two steps. First, a variation of a 'World Café' will be used to initiate and lead a collective reflection around the AA pillars and definition identified by the research team. A World Café is a simple yet powerful method to enable meaningful conversations driven by participants and the topics that are relevant and important to them, ${ }^{35}{ }^{36}$ to lay the groundwork for common understandings. Building on the results of the World Café, a 'carousel brainstorming' technique will be used with AA experts to brainstorm on important components to be included in each pillar. A Carousel Activity is a communicative and interactive opportunity for participants to get up and move around a room in a circular fashion, stopping intermittingly to comment, discuss or respond in writing to probing headings/questions/topics/themes posted by a facilitator. ${ }^{37}$ This technique allows for small group discussion, followed by whole-group collective reflection. ${ }^{38}$ Facilitation techniques ensure that everyone is able to participate equally and is able to express his/her viewpoint freely, while ignoring hierarchical concerns. ${ }^{39}$ The results of this meeting will be analysed by the research team to come to a consensus on the conceptual model of AA including the name, number of pillars and definition of each identified pillar. This will serve as groundwork for the e-Delphi consultation.

\section{Phase 2: creation of the AA reflective tool through e-Delphi consultation}

Phase 2 will be conducted on an online survey tool (Survey Monkey platform). After each round, a personalised report will be sent to each AA expert providing an overall view of responses as well as their own, in an anonymised format. A list of processes to operationalise the various pillars and subpillars developed during phase 1 will be reviewed and adapted by the research team before submission to the expert panel. The mandate of the panel will be to set the importance of the suggested processes for each of the subpillars and to achieve a consensus on a final list of processes, considered to be very important or essential for assessment of an AA practice. Experts will also be surveyed regarding the adequacy of suggested response scales for each item of the reflective AA tool.

\section{Round 1}

An individualised link to a personalised questionnaire will be sent to each AA expert. The first round of consultation will propose subpillars that have emerged from phase 1. For each of the proposed subpillars, AA experts will be asked to rate their level of agreement (on a scale of 1-9) regarding the relevance of this subpillar to the concept of AA. The median as well as measures of dispersion will be used as indicators of the level of consensus. There is no commonly defined rule to determine achievement of consensus, so a pre-hoc decision was made to consider $75 \%$ agreement to be consensus. Based on applied methods to determine consensus in a Delphi, ${ }^{40}$ we define the following three zones: a median between 7 and 9 indicates high relevance, a median between 1 and 3 indicates low relevance and a middle zone relevance of a median between 4 and 6 where the relevance is uncertain. These 
subpillars will be retained for a second round of consultation. A 9-point evaluation scale was chosen for this phase to allow participants to express their perception of relevance of a subpillar using a wide range of possibilities. Experts will also be asked to provide their definition, to comment on the subpillar, to indicate if this subpillar is associated with the appropriate pillar and to add any subpillars they think are missing along with suggested definitions.

\section{Round 2 and 3 surveys}

A feedback report from the previous round will accompany each new round of questionnaires, including the level of consensus achieved along with global and individual relevance scores for each item. In rounds 2 and 3 (and further if needed), the importance of the suggested items will be rated using 5-point Likert scale. The specification of each element of the response scale (1=Not important at all, 2=Little important, 3=Somewhat important, 4=Very important and $5=$ Essential) is intended to simplify the respondents' burden of response while adding clarity to the responses obtained. Consensus will be attained if $75 \%$ of respondents rate an item 'Very important' or 'Essential.' More specifically, consensus will be reached with a median rating of 4 or more, with an IQR of less than 1 . If an item is rated below 4 by more than $25 \%$ of respondents, this be interpreted to be non-consensus. AA experts will be given the opportunity to modify their initial response in light of the answers provided from the group, so as to facilitate the group evolution towards consensus. ${ }^{33} 42$ They will also be asked to score new propositions and modifications emerging from the previous round. The process will continue with further rounds until a consensus on the importance of each item is reached or not. ${ }^{3342}$ The e-Delphi rounds will cease when an acceptable degree of consensus is reached. ${ }^{43}$ Particular attention will be given following each round to assess whether consensus has been reached over a particular round, or rather evolved throughout the process, and whether the group's opinion has changed over the rounds. ${ }^{45}$ Phase 2 will result in the creation of the online reflective tool based on the list of items for which consensus was achieved, to rigorously assess the processes required in an AA practice.

\section{Phase 3: assessment and applicability of the newly developed AA reflective tool and development of a Repository of recommendations}

A subgroup of 5-10 AA experts who participated in phases 1 and/or 2 will be consulted to formulate and prioritise recommendations for an optimal AA practice.

\section{AA reflective tool refinement}

Survey completion sessions will be organised with PHC professionals and staff from five different PHC clinics, who will not have been involved in the previous phases of the study. These survey completion sessions will include feedback discussions on the completion of the tool, and will be led by the research team. Following cognitive testing techniques, ${ }^{46}$ these sessions are intended to identify items that are not clear or need to be reformulated, as well as any difficulties encountered during completion of the questionnaire. Following this piloting and development period, the AA reflective tool will be considered to be final, and ready to be evaluated by a larger number of potential users.

\section{Development of a repository of recommendations}

A repository of recommendations aligned with the different components of the revised AA model will be created, in relation to the result obtained after the completion of the tool. This repository of recommendations will be made available through the electronic platform, on completion of the evaluation using the AA reflective tool. Recommendations will be personalised according to the professional 'portrait' based on their score on each pillar, providing them with actionable avenues. Such an approach was inspired by the primary care quality improvement tool of the College of Family Physicians of Canada. ${ }^{27}$

The repository of recommendations will be inspired by systematic collation of best practices, by reviewing the literature related to each of the components of AA (eg, improving interprofessional collaboration, optimisation of telephone reception, managing escalation of emergencies) and using feedback generated by the expert panel on the final e-Delphi round. Implementation guides as well as locally developed help tools will serve as sources of recommendations for the repository and will be expanded with experiences of AA experts and their close collaborators. The repository will be expanded and refined during the third round of the e-Delphi and during the survey completion sessions. If discussion of recommendations cannot be addressed in the third round of the e-Delphi, we will bring the discussion to experts in an additional face-to-face or virtual meeting.'

\section{Assessing the psychometric properties of the reflective tool}

The final step of development of the tool will consist of the evaluation of some of its psychometric properties. To do so, we plan to recruit a minimum of 150-200 PHC professionals in at least $10 \mathrm{PHC}$ clinics that have not been involved in the development of the tool. ${ }^{47}$ The family physicians, nurses and other professionals working in those PHC clinics will be asked to complete the newly developed tool and comment on its content. Following qualitative feedback, the first analysis will be at the item level: after excluding items with more than $4 \%$ missing values, we will do an item discrimination analysis to learn how well an item can discriminate between high and low AA performers. Items with a lack of variation in responses-that is, that are either too easy to or too difficult to attain-will be reviewed for content and adequacy of the response scale. Other properties tested will be the tool's reliability (repeatability and intra-reliability and inter-reliability in different contexts or between different types of healthcare professionals), and validity (construct validity with a confirmatory factor analysis). Finally, 
internal consistency will be analysed for each pillar and subpillar.

\section{Patient and public involvement}

At least two patients will be invited to the face-to-face meeting in phase 1 as well as the e-Delphi survey. We also intend to consult the patient partners' group related to our research infrastructure at the end of the tool development to discuss issues that may have arisen and could require a patient point of view. This group is composed of five patient partners involved in different research project on AA.

\section{Ethics and dissemination}

Ethics and consent for participation will be sought at each phase of the study. Participation in a meeting or completion of an electronic survey will be considered to be consent for participation and use of anonymised data; as in focus groups or individual interviews, a written consent will be sought.

The results of dissemination plan includes communications through the PHC community including our partner organisations, and to the scientific community via the peer-reviewed literature and conferences. We will attempt to reach many PHC professionals to let them know of our findings through professional organisations and by organising a symposium that will bring together our expert participants as well as colleagues from their organisations or from other organisations and PHC clinics. As so, elements and lessons learnt from this study will be shared through multiple community media resources, including presentations and webinars, newsletters and a symposium on AA initiated by the research team.

\section{DISCUSSION}

Scientific articles on the foundations of AA have been published over the past 20 years. During this time interdisciplinary collaborative practice has evolved within PHC family practices, with several healthcare professionals now being part of the PHC team. This study will help to redefine the foundations of the AA model by integrating an interdisciplinary team-based focus, while considering changes that have been put in place in PHC practices. The inclusion of various PHC stakeholders in the tool development process will allow the tool and its content to reflect realities experienced in the field. Participation of AA champions in the overall development process of the AA reflective tool will benefit the community's acceptance of the tool.

This study will provide a rigorously developed up-todate AA reflective tool based on the literature and on the experiences of various PHC stakeholders, while providing a response to their expressed needs for a reflective tool. The newly developed reflective tool will be helpful as the Ministry of Health and Social Services and professional medical organisations are currently promoting AA in PHC settings and other professional associations across
Canada. Timely access is a key pillar of PCMH, a wellknown model to guide the development of PHC around the world. The reflective tool on AA developed in this study will be available for use and adaptation in different countries.

The repository of recommendations developed in parallel with the tool will provide the AA tool users with advice in relation with their own AA evaluation, and provide pragmatic and personalised recommendations to improve their AA practice. This will all be conveniently available online.

\section{Author affiliations}

${ }^{1}$ Faculty of Medicine and Health Sciences, Department of Community Health, University of Sherbrooke, Longueuil, Quebec, Canada

${ }^{2}$ Department of Family Medicine and Emergency Medicine, Universite de

Sherbrooke Faculte de medecine et des sciences de la sante, Longueuil, Quebec, Canada

${ }^{3}$ Nursing Faculty, Université Laval, Quebec, Quebec, Canada

${ }^{4}$ Faculty of Medicine and Health Sciences, University of Sherbrooke, Longueuil, Quebec, Canada

${ }^{5}$ School of Nursing Sciences, University of Sherbrooke, Longueuil, Quebec, Canada ${ }^{6}$ Faculty of Medicine and Health Sciences, Université de Sherbrooke, Sherbrooke, Quebec, Canada

${ }^{7}$ Université de Montréal Faculté de Médecine, Montréal, Québec, Canada

${ }^{8}$ École nationale d'administration publique — ENAP, Université du Québec à

Montréal, Montreal, Québec, Canada

${ }^{9}$ Université de Montréal, Montreal, Québec, Canada

${ }^{10}$ Faculty of Medicine, McGill University, Montreal, Québec, Canada

Twitter Mylaine Breton @bretonmylaine, Sabina Abou Malham @sabmalham and Lara Maillet @lara_maillet

Acknowledgements The authors thank Meg Sears for scientific and linguistic editing.

Contributors MB and IG led the conceptualisation and design of the study, but all authors were involved. MB, IG and CB will lead the coordination of the study. MB, IG and CB wrote the first draft and all authors critically reviewed it and provided comments to improve the first draft and revised version of the manuscript. All authors read and approved the final version.

Funding This study received a two years funding from the Ministry of Health and Social Services of Quebec (MSSS) of $\$ 500000$. The funding organisation was not involved in the design of the study nor writing of the protocol (980475).

Competing interests None declared.

Patient and public involvement Patients and/or the public were involved in the design, or conduct, or reporting or dissemination plans of this research. Refer to the Methods section for further details.

Patient consent for publication Not applicable.

Provenance and peer review Not commissioned; externally peer reviewed.

Open access This is an open access article distributed in accordance with the Creative Commons Attribution Non Commercial (CC BY-NC 4.0) license, which permits others to distribute, remix, adapt, build upon this work noncommercially, and license their derivative works on different terms, provided the original work is properly cited, appropriate credit is given, any changes made indicated, and the use is non-commercial. See: http://creativecommons.org/ licenses/by-nc/4.0/.

\section{ORCID iDs}

Mylaine Breton http://orcid.org/0000-0001-5713-9618

Isabelle Gaboury http://orcid.org/0000-0002-2881-4714

Sabina Abou Malham http://orcid.org/0000-0003-2885-6807

Catherine Hudon http://orcid.org/0000-0001-6140-9916

Lara Maillet http://orcid.org/0000-0001-6192-183X

Nadia Deville-Stoetzel http://orcid.org/0000-0001-7387-6511 


\section{REFERENCES}

1 Ansell D, Crispo JAG, Simard B, et al. Interventions to reduce wait times for primary care appointments: a systematic review. BMC Health Serv Res 2017;17:295.

2 Collège des médecins de famille du Canada, Une vision pour le Canada. La pratique de la médecine familiale: Le centre de médecine de famille. Mississauga, on, 2011. Available: https://www.google. $\mathrm{com} /$ url? sa=t\&rct=j\&q=\&esrc=s\&source=web\&cd=\&cad=rja\&uact= 8\&ved=2ahUKEwjyqb075rjqAhU1YTUKHSQPDNcQFjACegQIAxAB\& url=https\%3A\%2F\%2Fwww.cfpc.ca\%2FuploadedFiles\%2FAbout Us\%2FFM-Professional-Profile-FR.pdf\&usg=AOvVaw3wJIJ3K5ttqlxd otLSRz5L

3 Murray M, Berwick DM. Advanced access: reducing waiting and delays in primary care. JAMA 2003;289:1035-40.

4 Abou Malham S, Touati N, Maillet L, et al. What are the factors influencing implementation of advanced access in family medicine units? A Cross-Case comparison of four early Adopters in Quebec. Int J Family Med 2017;2017:1-15

5 Bundy DG, Randolph GD, Murray M, et al. Open access in primary care: results of a North Carolina pilot project. Pediatrics 2005;116:82-7.

6 Rose KD, Ross JS, Horwitz LI. Advanced access scheduling outcomes: a systematic review. Arch Intern Med 2011;171:1150-9.

7 Fournier J, Heale R, Rietze LL. I can't wait: advanced access decreases wait times in primary healthcare. Healthc Q 2012;15:64-8.

8 Hudec JC, MacDougall S, Rankin E. Advanced access appointments: effects on family physician satisfaction, physicians' office income, and emergency department use. Can Fam Physician 2010;56:e361-7.

9 Rivas J. Advanced access scheduling in primary care: a synthesis of evidence. J Healthc Manag 2020;65:171-84.

10 Belardi FG, Weir S, Craig FW. A controlled trial of an advanced access appointment system in a residency family medicine center. Fam Med 2004;36:341-5.

11 Bennett CC. A healthier future for all Australians: an overview of the final report of the National health and hospitals reform Commission. Med J Aust 2009;191:383-7.

12 Ahluwalia S, Offredy M. A qualitative study of the impact of the implementation of advanced access in primary healthcare on the working lives of general practice staff. BMC Fam Pract 2005;6:39.

13 College of Family Physicians of Canada. Best Advice- timely access to appointments in family practice, 2012. Available: https://portal. cfpc.ca/ResourcesDocs/uploadedFiles/Health_Policy/_PDFs/2012 Final Best Advice_Enhancing_Timely_Access.pdf

14 Lemire F. Refreshing the patient's medical home: new vision for providing exceptional care in family practice. Can Fam Physician 2019;65:152.

15 Gaboury I, Breton M, Perreault K, et al. Interprofessional advanced access - a quality improvement protocol for expanding access to primary care services. BMC Health Serv Res 2021;21:812.

16 Breton M, Maillet L, Paré I, et al. Perceptions of the first family physicians to adopt advanced access in the province of Quebec, Canada. Int J Health Plann Manage 2017;32:e316-32.

17 Breton M, Maillet L, Duhoux A, et al. Evaluation of the implementation and associated effects of advanced access in University family medicine groups: a study protocol. BMC Fam Pract 2020;21:41.

18 Brazeau S, Couture P, Karemere-Bimana H. Guide pour l'implantation de l'accès adapté: l'expérience d'une région: Laval. Laval: Centre intégré de santé et de services sociaux de Laval, 2016. http://www. santecom.qc.ca/Bibliothequevirtuelle/Laval/9782550744337.pdf

19 Centre intégré de santé et de services sociaux du Bas Saint-Laurent. Outils, zone professionnelle, Accès Adapté, 2020. Available: https:// www.cisss-bsl.gouv.qc.ca/zone-professionnelle/acces-adapte/outils

20 Health Quality Ontario. Quality improvement e-learning modules: timely access to primary care. hqontario.ca, 2020. Available: https:// www.hqontario.ca/Quality-Improvement/E-Learning-and-Events/ELearning-Modules-Timely-Access-to-Primary-Care

21 Institute of Medicine (U.S.), Kaplan G, Lopez MH, et al., editors. Transforming health care scheduling and access: getting to now. Washington, D.C: The National Academies Press, 2015.

22 et alLukas C, Meterko M, Mohr D. The implementation and effectiveness of advanced clinic access, 2004. Available: https:// www.researchgate.net/publication/228478818_The_Implementation and_Effectiveness_of_Advanced_Clinic_Access/citation/download

23 Institute for Health Improvement. Primary care access, 2020. Available: http://www.ihi.org/Topics/PrimaryCareAccess/Pages/ default.aspx
24 Practice Management Network. Improving acces, responding to patients. A 'how-to' guide for GP practices, 2009. Available: https:// www.choiceforum.org/docs/gpguide.pdf

25 Langley GJ, ed. The improvement guide: a practical approach to enhancing organizational performance. 2nd ed. San Francisco: Jossey-Bass, 2009.

26 Stenov V, Wind G, Skinner T, et al. The potential of a self-assessment tool to identify healthcare professionals' strengths and areas in need of professional development to aid effective facilitation of group-based, person-centered diabetes education. BMC Med Educ 2017;17:166.

27 The College of Family Physicians of Canada. The self-assessment tool for the Patient's Medical Home, 2018. Available: https:// patientsmedicalhome.ca/self-assess/

28 The Joint Learning Network for Universal Health Coverage. UHC primary health care self-assessment tool, 2015. Available: https:// www.jointlearningnetwork.org/wp-content/uploads/2019/11/UHCPHC-Self-Assessment_Tool.pdf

29 The American Medical Association. The caregiver self-assessment tool. Healthinaging.com: trusted information. better care, 2015. Available: https://www.healthinaging.org/tools-and-tips/caregiverself-assessment-questionnaire

30 Veugelers R, Gaakeer MI, Patka P, et al. Improving design choices in Delphi studies in medicine: the case of an exemplary physician multiround panel study with $100 \%$ response. BMC Med Res Methodol 2020;20:156.

31 Jünger S, Payne SA, Brine J, et al. Guidance on conducting and reporting Delphi studies (CREDES) in palliative care: recommendations based on a methodological systematic review. Palliat Med 2017;31:684-706.

32 Falzarano M, Pinto Zipp G, Pinto G. Seeking consensus through the use of the Delphi technique in health sciences research. $J$ Allied Health 2013;42:99-105.

33 Hsu C-C, Sandford BA. The Delphi technique: making sense of consensus. Practical Assessment, Research and Evaluation 2007;12:1-6.

34 Fédération des médecins omnipraticiens du Québec. Accès adapté, organisation de la pratique: L'accès adapté en un clic ! Fédération des médecins omnipraticiens du Québec, 2015. Available: https:// www.fmoq.org/pratique/organisation-de-la-pratique/acces-adapte/

35 SessionLab. Hyper island, 2020. Available: https://www.sessionlab. $\mathrm{com} / \mathrm{methods/world-cafe}$

36 The WorldCafe. World Cafe method, 2020. Available: http://www. theworldcafe.com/key-concepts-resources/world-cafe-method/

37 Delgado RA. Carousel activity protocol. Utica College, 2020. Available: https://www.uticaschools.org/site/handlers/filedownload. ash $x$ ? moduleinstanceid $=\% 20273 \&$ dataid $=286 \&$ FileName $=$ Carousel\%20Activity\%20Protocol.pdf

38 Simon CA. Strategy guide: brainstorming and reviewing using the Carousel strategy. ReadWriteThink, 2020. Available: http://www. readwritethink.org/professional-development/strategy-guides/ brainstorming-reviewing-using-carousel-30630.html

39 Liberating Structures. Liberating structures, 2020. Available: http:// www.liberatingstructures.com/

40 et alFitch K, Bernstein SJ, Aguilar MD. The RAND/UCLA Appropriateness method users' manual, 2001. Available: https:// apps.dtic.mil/dtic/tr/fulltext/u2/a393235.pdf

41 Dionne CE, Tremblay-Boudreault V. Chapitre 7 - L'APPROCHE DELPHI, Application dans la conception d'un outil clinique en réadaptation au travail en santé mentale. In: Méthodes qualitatives, quantitatives et mixtes. 2e édition. Québec, Qc, Canada: Presses de I'Université du Québec, 2020. ISBN: 978-2-7605-5139-8. https:// www.puq.ca/catalogue/livres/methodes-qualitatives-quantitativesmixtes-edition-3773.html

42 Hsu C-C, Sandford B. Minimizing non-response in the Delphi process: how to respond to non-response. Practical Assessment, Research, and Evaluation 2007;12:17.

43 Skulmoski G, T J, Hartman F, Krahn J. The Delphi method for graduate research. JITE:Research 2007;6:001-21.

44 de Villiers MR, de Villiers PJT, Kent AP. The Delphi technique in health sciences education research. Med Teach 2005;27:639-43.

45 Holey EA, Feeley JL, Dixon J, et al. An exploration of the use of simple statistics to measure consensus and stability in Delphi studies. BMC Med Res Methodol 2007;7:52.

46 Willis GB. Analysis of the cognitive interview in questionnaire design. Oxford University Press: Oxford:, 2015.

47 Martin CR, Hollins Martin CJ. Minimum sample size requirements for a validation study of the birth satisfaction scale-revised (BSS-R). Journal of Nursing and Practice 2017;1:25-30. 